\section{The Influence of Cytoflavin Therapy on the Cerebral Hemodynamics Condition in Patients with Various Stages of Hypertensive Disease}

Belova Liudmila Anatolyevna ${ }^{1 *}$ Kolotik-Kameneva Olesya Yurievna ${ }^{2}$, Mashin Viktor Vladimirovich ${ }^{1}$, Belova Natalya Vyacheslavovna $^{3}$, Angelo Scuderi ${ }^{4}$ and Pier Luigi Antignani ${ }^{5}$

${ }^{1}$ Department of Neurology, Neurosurgery, Physiotherapy and Physical therapy, Federal State Budget Financed Educational Institution of Higher Professional Education, Ulyanovsk State University, Ulyanovsk, Russia

${ }^{2}$ Neurology Department for Patients with a Stroke, Central Clinical Medical Sanitary Unit, Ulyanovsk, Russia

${ }^{3}$ Scientific Center of Neurology, Moscow, Russia

${ }^{4}$ Department of Angiology and Vascular Surgery, University Hospital Santa Lucinda, Sorocaba, Brazil

${ }^{5}$ Vascular Center of Nuova Villa Claudia, Rome, Italy

\begin{abstract}
Objective

To investigate the effect of cytoflavin on cerebral hemodynamics at various stages of arterial hypertension.

Materials and methods

One hundred forty patients with hypertension of I-III stages were divided into two groups taking into account their stratification:

I. The patients of treatment group 1 received a comprehensive therapy consisting of antihypertensive therapy and a metabolic drug, cytoflavin $10 \mathrm{mg}$ from the first to the tenth day by an intravenous drop infusion, then for 60 days - they received 2 tablets in a dose of $320 \mathrm{mg}$ twice a day

II. The patients of treatment group 2 - received antihypertensive therapy only

III. The control group was composed of 30 apparently healthy people.
\end{abstract}

*Corresponding author: Belova Liudmila Anatolyevna, Department of Neurology, Neurosurgery, Physiotherapy and Physical therapy, Federal State Budget Financed Educational Institution of Higher Professional Education, Ulyanovsk State University, Ulyanovsk, Russia, Tel: +7 9084905015; E-mail: labelova@mail.ru

Citation: Belova LA, Kolotik-Kameneva OY, Mashin VV, Belova NV, Scuderi A, et al. (2016) The Influence of Cytoflavin Therapy on the Cerebral Hemodynamics Condition in Patients with Various Stages of Hypertensive Disease. J Non Invasive Vasc Invest 1: 001.

Received: May 26, 2016; Accepted: August 02, 2016; Published: August 15, 2016
All the patients were examined clinically and instrumentally before the therapy administration and in 70 days after the beginning of treatment. The state of cerebral hemodynamics was assessed with the use of a comprehensive ultrasonic examination algorithm of the cerebral vascular system based on the concept that it is composed of five functional and morphological levels.

Results

The signs of cerebral vascular remodeling are observed in patients with hypertension which are characterized by a change of PI, RI values, intima media complex, the decrease of blood flow in the common carotid arteries, internal arteries, vertebral and medial cerebral arteries, by the decrease of Rosenthal veins reactivity, blood flow decrease in Rosenthal veins, the internal jugular veins and increase of blood flow in the vertebral veins in stages I-III of the disease.

A complex therapy with application of cytoflavin is accompanied by blood flow increase in the common carotid arteries, internal carotid arteries, medial cerebral arteries in the first stage of the disease; in vertebral arteries in stages I-II of the disease it is accompanied by the improvement of Rosenthal vein reactivity, the restoration of the blood flow values to control ones in Rosenthal veins, internal jugular veins and also by the blood flow decrease in vertebral veins while examining a patient in recumbent position.

\section{Conclusion}

Changes of cerebral hemodynamics have been found in all structural and functional levels of the vascular system in the brain beginning with hypertension stage I. A complex therapy with application of cytoflavin was administered in the following regimen: an intravenous drop infusion of $10 \mathrm{mg}$ from day 1 to day 10 , then for 60 days the patients received 2 tablets twice a day that lead to the abatement of marked manifestations of cerebral arterial insufficiency at the incipient stages of the disease, microcirculatory disorders and venous insufficiency in stages I-III of the disease.

The obtained results have shown the need of investigating cerebral haemodynamics at five structurally functional levels of the cerebrovascular system and allow us to recommend cytoflavin for use in a complex therapy of patients with arterial hypertensive from the first stage.

Keywords: Cerebral hemodynamics; Cytoflavin; Hypertension; Vascular remodeling

\section{Introduction}

At present the diseases of the blood circulatory system, hypertension being one of them, occupy a considerable place in fatal outcome causes in a great number of economically developed countries of the world [1-5]. With age the incidence of this disease becomes high and by the age of 60 it affects $30-50 \%$ of the population [6]. Progression of hypertension is accompanied not only by the heart lesions. The vascular system of the brain is also affected with a subsequent formation of a complicated and diverse set of primary destructive and secondary reparative changes and adaptive processes on all the structural and functional levels of the vascular system of the brain $[1,7,8]$. Recalibration of the arteries leads to a reduction of the cerebral blood flow in cortical segments, to ischemia of the brain tissue [9-11]. The decrease of the brain vessel responsiveness is also considered as an 
important prognostic factor in relation to progression of blood supply insufficiency of the brain $[7,11,12]$. It has been proved that cerebral venous discirculation is associated with development and progression of hypoxic-ischemic brain cell damage, microcirculatory disorders, reduction of the hemodynamic reserve of the brain and its metabolic disorder [13-17]. All these changes trigger the development of cerebral and focal neurological symptoms with the risk of severe complications that lead to social and household maladaptation and disability of a patient.

The goal of searching for the most suitable methods of diagnostics and elimination of cerebral vascular remodeling manifestations in case of hypertension is very important when we take into account an underlying influence of cerebral hemodynamics disorders on a clinical picture of the disease and its prognosis. Cytoflavin, a drug produced in Russia is one of the drugs that proved its efficacy in multi-center placebo-controlled studies conducted earlier in the treatment of cardio-vascular diseases and their complications [10]. Cytoflavin represents a complex substance of known and widely-used metabolites: succinic acid in $\mathrm{Na}, \mathrm{N}$ - metylglucammonia succinate, riboxinum and two coenzymes - riboflavin and nicotinamide. This medication has a positive effect on the processes of energy formation in a cell reducing the intensity of oxidative stress and suppressing an excessive release of excitatory neurotransmitters in case of ischemia.

The purpose of the study was to investigate the influence of cytoflavin on the cerebral haemodynamics at various stages of hypertension.

\section{Materials and Methods}

A prospective cohort study was conducted at the neurology department of the Central medical and sanitary unit in the city of Ulyanovsk. The study included 140 patients with hypertension stages I-III, 38 (27.1\%) males and 102 (72.7\%) females at the age from 47 to 73 years (the mean age of patients was $62.1 \pm 10.7$ years).

The duration of arterial hypertension in all the patients involved in the study was more than 5 years $(7.3 \pm 4.8$ years $)$. The diagnosis, degree and stage of hypertension were verified in view of the recommendations given by All Russian Scientific Society of Cardiologists in 2013. Fifty - three (37.9\%) patients had arterial hypertension of the first degree, 50 patients $(35.71 \%)$ - arterial hypertension of degree II and 37 patients (26.4\%) - arterial hypertension of degree III (recommendations of All Russian Scientific Society of Cardiologists, 2013). The criteria to include the patients in the study were: hypertension of stages I, II, III. The criteria for exclusion from the study were: psychic, endocrine, hematologic, oncological, autoimmune, infectious diseases; a cerebral stroke in an acute period and rehabilitation period up to 1 year, organic disease of the brain, a cranial and cerebral trauma; cardiac insufficiency decompensation, secondary (symptomatic) arterial hypertension; heart rhythm disorder and cardiac conduction disorder; pregnancy, breastfeeding, a transient ischemic attack or hypertensic crisis at the moment of inclusion in the study; taking antioxidant or nootropic drugs during the last 3 months.

All patients with hypertension stages I-III included in the study were randomized by means of the sealed envelopes method and divided into two groups in view of their stratification. The patients of Treatment Group 1 (TG 1), 74 people (27 patients with hypertension stage I, 27 - patients with hypertension stage II, 20 - with hypertension stage III) received a combination treatment consisting of antihypertensive therapy and application of cytoflavin (Polisan, Russia) in the following treatment regimen: intravenous drop infusion of $10 \mathrm{mg}$ from day 1 to day 10 , then for 60 days - 2 tablets in a dose of $320 \mathrm{mg}$ twice a day; the patients of Treatment Group 2 (TG 2), 66 people (24 patients with hypertension stage I, 24 - with hypertension stage II, 18 - with hypertension stage III) - antihypertensive treatment which included hypotensive medications (monotherapy in the form of APF inhibitors $(6.7 \pm 2.1 \mathrm{mg} /$ days $)$, thiazid-like diuretics $(2.5 \mathrm{mg} /$ days $), \mathrm{B}$-adrenoblockers $(31.6 \pm 7.8 \mathrm{mg} /$ days $)$ or antagonists of calcium $(9.7 \pm 4.5 \mathrm{mg} /$ days $)$ at $\mathrm{AH}$ of degree $\mathrm{I}$, a combination of APF inhibitors (19.4 $\pm 5.8 \mathrm{mg} /$ days) with thiazid-like diuretics $(2.5 \mathrm{mg} /$ days $)$, APF inhibitors $(19.1 \pm 4.6 \mathrm{mg} /$ days $)$ with B-adrenoblockers (36.4 $\pm 9.3 \mathrm{mg} /$ days $)$, APF inhibitors $(19.7 \pm 6.1$ $\mathrm{mg}$ /days) with thiazid-like diuretics $(2.5 \mathrm{mg} /$ days $)$ and B-adrenoblockers $(25.6 \pm 9.8 \mathrm{mg} /$ days $)$ at degree $\mathrm{AH}$ II-III) in combination with a low-salt diet. All the patients before the observation had target values of arterial blood pressure. Control Group (CoG) included 30 apparently healthy people without statistically significant differences in age and sex distribution. All the patients were examined clinically and instrumentally before the treatment administration and after 70 days from the beginning of treatment.

To study cerebral hemodynamics, a special procedure of examining the vascular system of the brain with Intravascular Ultrasound (IVUS) was applied taking into consideration the concept that the vascular system consists of five functional and morphological levels. The first structural and functional level comprises major arteries of the head - Common Carotid Arteries (CCA), Internal Carotid Arteries (ICA), Vertebral Arteries (VA). The second level includes Medial Cerebral Arteries (MCA); the third level - Microcirculatory Bloodstream (MCB); the fourth level - Rosenthal Veins (RV); the fifth level - Internal Jugular Veins (IJV), Vertebral Veins (VV). The examination of the major arteries of the head, main veins and cerebral hemodynamics was performed with ultrasonic scanners Viamo SSA-640A (Toshiba, Japan) and a linear array probe with a center frequency of $7.5 \mathrm{MHz}$.

Vessel scanning of the first and fifth structural and functional levels of the vascular system of the brain was carried out in the transverse and longitudinal planes. The internal jugular vein was examined throughout its length (from the corner of the lower jaw to the bulb), quantitative doppler values were registered $3 \mathrm{~cm}$ below the level of the facial vein junction. Competence of vessels, their diameter, the presence of deformations or changes in the vessel were assessed as well as the mobility of a vascular wall, the condition of perivascular tissues, Linear Blood Flow Velocity (LBFV), minimum Velocity (Vmin), time-averaged mean Velocity (Vmed), maximum Velocity (Vmax); Index of Resistance (IR) and Pulsation Index (PI); maximum volumetric blood flow Velocity (Vvolmax).

Measurement of the thickness of an intima media complex was taken 1-1.5 cm proximal of the bifurcation of the common carotid arteries on the posterior wall of the artery. There were no patients with occluded common carotid arteries in our study.

Examination of the vessels of the second and fourth morpho-functional levels was carried out with Transcranial Duplex Scanning (TDS), with the use of ultrasonic scanners Viamo SSA-640A (Toshiba, Japan) and an array probe with a center frequency of $2.5 \mathrm{MHz}$. Linear blood flow velocity, resistance index, pulsation index in the medial cerebral arteries, linear blood flow velocity in Rosenthal veins were assessed. 
To examine the vessels of the third structural and functional level - MCB - the cerebral vascular reserve was assessed by studying Rosenthal veins reactivity. To this end, a test with orthostatic loading was carried out on the standing frame at $60-80$. The test was considered to be positive in the presence of the statistically significant decrease of velocity values in orthostasis in comparison with baseline values.

Statistical processing of research results was carried out by means of the Statistica 8.0 program to find the significance level (p). Depending on the type of distribution, the method of percent comparison and non-parametric methods (Mann-Whitney U-criterion, Wilcoxon criterion, Spirmen correlation analysis) were used. Results were presented as the median, 25 and $75 \%$ quartiles, $\mathrm{M}(25 \%$; $75 \%)$. Differences with error probability less than $5 \%$ of the first kind were considered to be statistically significant $(\mathrm{p}<0.05)$.

\section{Results}

The main arteries of the head have a paramount significance in blood supply of the brain [8]. We assessed structural and functional changes of the extracranial arteries.

The analysis of the structural findings and the values characterizing the Peripheral Vascular Resistance (PVR) of common carotid arteries has revealed the changes that are typical of hypertension: the vessel motion trajectory alteration, defect of the intima media complex structure, an increase in the thickness of intima media complex in hypertension stage III $(1.0(0.8 ; 1.1)$ in comparison with the control group $(0.7(0.5 ; 0.8), \mathrm{p}<0.05)$, increase of PI (1.63 (1.37; $1.89)$ ) in hypertension stage III in comparison with the control group (1.58 $(1.46 ; 1.62), \mathrm{p}<0.03)$. Over time on the 70th day the blood flow values did not change in any group.

Velocity values of blood flow in Common Carotid Arteries (CCA) were studied in patients with hypertension (Figures 1 and 2).

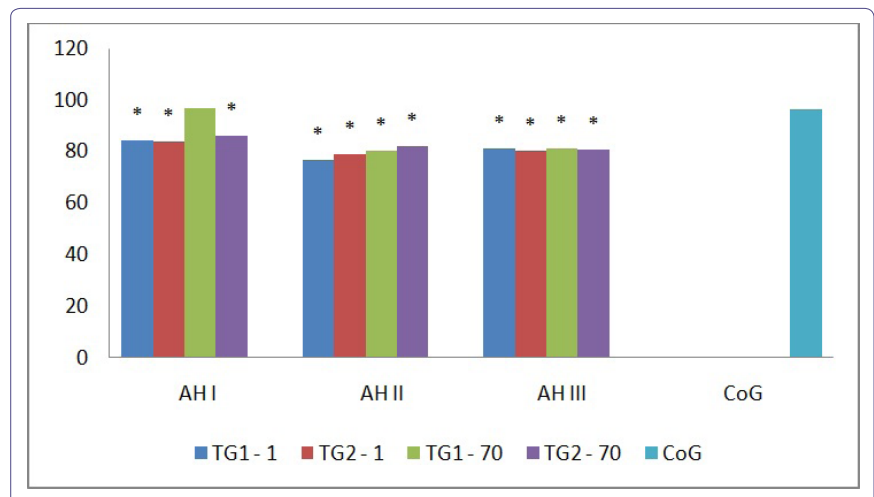

Figure 1: Dynamic Vmax pattern $(\mathrm{cm} / \mathrm{s})$ of common carotid arteries in patients with hypertension, ${ }^{*}-p<0.05$ - statistically significant differences in comparison with the control group.

The decrease of Vmax, Vvolmed $(81.5(65.9 ; 90.7) \mathrm{cm} / \mathrm{s}, 236.8$ (159.4; 301.7 ) $\mathrm{ml} / \mathrm{min}$ respectively) values was found in common carotid arteries in treatment group 1 and in treatment group 2 compared with the control group in all hypertension stages $((96.7$ $(85.7 ; 115.9) \mathrm{cm} / \mathrm{s}, 263.9(180.2 ; 338.6) \mathrm{ml} / \mathrm{min}$ respectively; $\mathrm{p}<0.05)$. On day 70 in the group that received a combined therapy with application of cytoflavin these values reached the values of the control group in stage I ( $\mathrm{p}>0.05)$ (Figures 1 and 2$)$.

Hemodynamic values of the internal carotid arteries were studied (Figure 3).

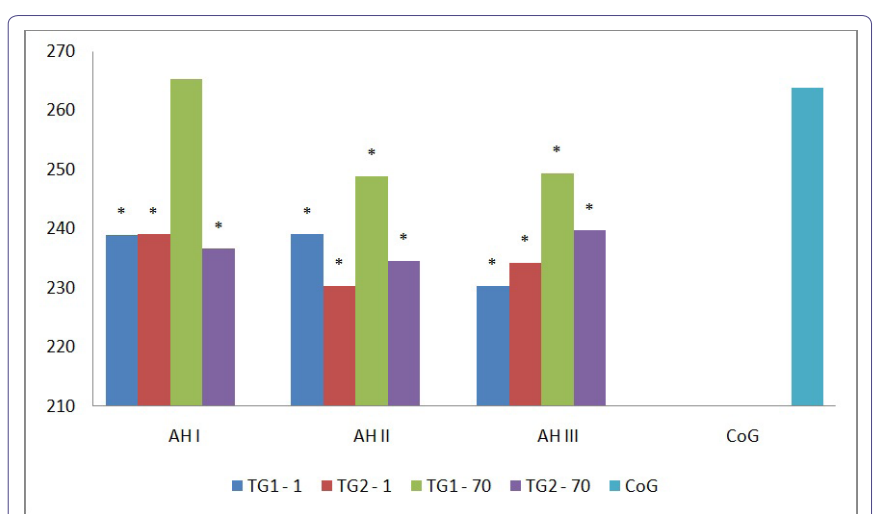

Figure 2: Dynamic Vvolmed pattern $(\mathrm{ml} / \mathrm{min})$ of common carotid arteries in patients with hypertension, ${ }^{*}-p<0.05$ - statistically significant differences in comparison with the control group.

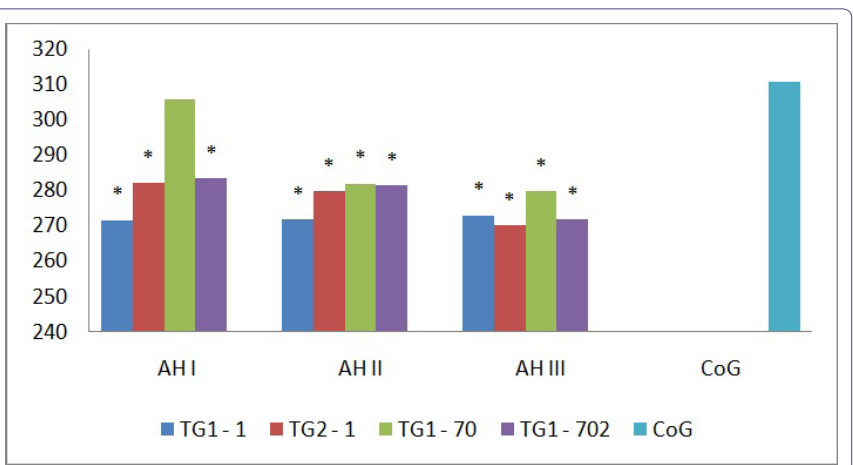

Figure 3: Dynamic Vvolmax pattern $(\mathrm{ml} / \mathrm{min})$ of internal carotid arteries in patients with hypertension, ${ }^{*}-p<0.05$ - statistically significant differences in comparison with the control group.

It was found that the decrease of values in Vmax, Vmin и Vvolmax $(70.4(64.7 ; 77.4) \mathrm{cm} / \mathrm{s}, 23.7(18.5 ; 27.8) \mathrm{cm} / \mathrm{s}$ и $274.8(176.2 ; 352.1)$ $\mathrm{ml} / \mathrm{min}$ respectively) independent of a hypertension stage in all the groups compared with the control group $(83.7(75.2 ; 90.9) \mathrm{cm} / \mathrm{s}, 32.4$ $(27.6 ; 41.9)$ см/с и $310.7(200.6 ; 405.2)$ мл/мин respectively; $\mathrm{p}<0.03)$. Over time in the group that received a combined therapy with application of cytoflavin the values of Vvolmax reached control values (305.8 (202.7; 384.9) $\mathrm{ml} / \mathrm{min} ; \mathrm{p}>0.05)$ in hypertension stage $\mathrm{I}$. Velocity values did not change in the group that received antihypertensive therapy only (Figure 3).

The analysis of blood flow velocity values was conducted in patients with hypertension of vertebral arteries (Figures 4 and 5).

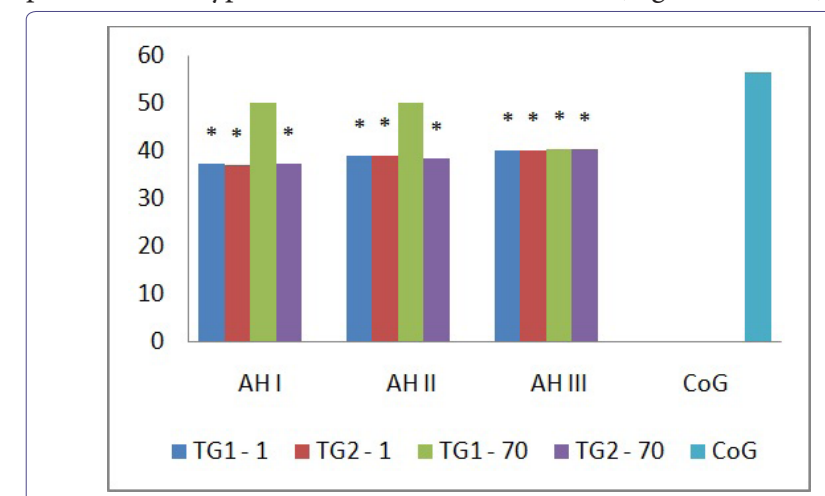

Figure 4: Dynamic Vmax pattern $(\mathrm{cm} / \mathrm{s})$ of vertebral arteries in patients with hypertension, ${ }^{*}-p<0.05$ - statistically significant differences in comparison with the control group. 


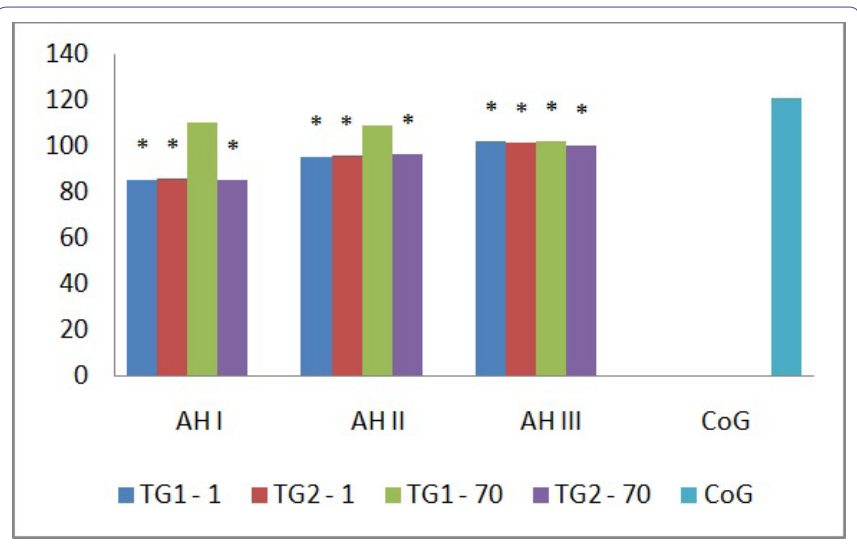

Figure 5: Dynamic Vvolmax pattern $(\mathrm{ml} / \mathrm{min})$ of vertebral arteries in patients with hypertension, ${ }^{*}-p<0.05$ - statistically significant differences in comparison with the control group.

In vertebral arteries the values of Vmax and Vvolmax (39.1 (31.4; $42.9) \mathrm{cm} / \mathrm{s}$, и $94.7(41.6 ; 133.8) \mathrm{ml} / \mathrm{min}$ respectively) in patients with hypertension stages I-III were lower than the control values (56.4 $(51.4 ; 62.3) \mathrm{cm} / \mathrm{s}, 120.4(61.4 ; 184.4) \mathrm{ml} / \mathrm{min}$ respectively; $\mathrm{p}<0.02)$ in both groups and on day 70 of therapy in treatment group 1 they became comparable to the values of the control group $s(\mathrm{p}>0.05)$ at stages I-II. In treatment group 2 the values remained the same (Figures 4 and 5).

While studying the values that characterize peripheral vascular resistance in medial cerebral arteries on day 1 and day 70 the increase of IR in stage I was revealed $(0.61[0.57 ; 0.65])$ in comparison with the control group (0.57 [0.31; 0.83], $\mathrm{p}>0.05)$.

Assessing the linear blood flow velocity in the medial cerebral arteries its decrease was found on values of Vmax, Vmin, Vmed $(106.3[89.7 ; 123.9] \mathrm{cm} / \mathrm{s}, 49.2[39.6 ; 61.8] \mathrm{cm} / \mathrm{s}$ and $75.3[63.1 ; 82.6]$ $\mathrm{cm} / \mathrm{s}$ respectively) in all hypertension stages in comparison with the control group $(133.7[110.8 ; 155.2] \mathrm{cm} / \mathrm{s}, 62.5[52.3 ; 74.8] \mathrm{cm} / \mathrm{s}, 92.5$ $[86.4 ; 100.3] \mathrm{cm} / \mathrm{s}$ respectively), $(\mathrm{p}<0.02)$. In the course of combined therapy with application of cytoflavin these values (131.7 [111.5; $151.9] \mathrm{cm} / \mathrm{s}, 61.7[51.3 ; 72.1] \mathrm{cm} / \mathrm{s}, 80.0[70.1 ; 89.9] \mathrm{cm} / \mathrm{s}$ respectively) became comparable with control ones $(p>0.05)$ only at stage I. A similar dynamic pattern was not observed in treatment group 1.

Thus, with an ultrasonic examination of intra and extracranial arterial bed in all stages of hypertension the signs of cerebral arterial insufficiency were revealed which are expressed by the decrease of velocity values of common carotid arteries, internal carotid arteries, vertebral arteries, medial cerebral arteries. During combined therapy with cytoflavin the increase of the linear velocity values of medial cerebral arteries, linear and volumetric velocity values of common carotid arteries, internal carotid arteries was noted at stage I, of vertebral veins at stages I, II.

A microcirculatory disorder impedes the normal course of metabolic processes, contributes to hypoperfusion and development of dystrophic changes of the brain cells. Since the direct examination of small arteries and capillaries is not feasible technically - the Cerebrovascular Reserve (CVR) was assessed which is directly associated with capillary lesions and determined by the Rosenthal veins reactivity level.

While examining the intracranial venous bed before treatment the decrease was seen in baseline values of Vmax and Vmin of Rosenthal veins and Rosenthal veins reactivity in all the patients with hypertension in comparison with the control group ( $\mathrm{p}<0.001)$. In the course of treatment the values of Vmax and Vmin (16.0 [13.4; $18.7] \mathrm{cm} / \mathrm{s}$ and $12,3[9.7 ; 14.3] \mathrm{cm} / \mathrm{s}$ respectively) in treatment group 1 became comparable to the values of the control group (16.0 $[12.0 ; 20.2]$ and $12.8[8.8 ; 16.9]$ respectively), $\mathrm{p}>0.05)$ in all stages. A decrease of the linear blood flow velocity values in orthostasis was also found $(13.3[10.2 ; 16.4] \mathrm{cm} / \mathrm{s}$ and $7.4[5.1 ; 9.7] \mathrm{cm} / \mathrm{s}$ respectively, $(\mathrm{p}<0.002))$ in comparison with baseline values at all stages of hypertension. A similar pattern was not revealed in treatment group 2.

The internal jugular veins are known to be the main venous collecting basin in the human and an outflow in the vertebral veins in recumbent position in healthy people usually is 5-9\% [18]. Evaluating the condition of extracranial veins in case of hypertension we found a change of the values characterizing peripheral vascular resistance at hypertension stage III in treatment group 1 and treatment group 2 which was expressed by the IR increase of internal jugular veins (0.79 [0.57;0.92] and $0.77[0.56 ; 0.98]$ respectively) and vertebral veins $0.74[0.51 ; 0.97]$ and $0.75[0.44 ; 0.93]$ respectively) compared with the control group $(0.74$ [0.54; 0.93$], \mathrm{p}<0.04$ and 0.7 [0.5; 0.82$]$, $\mathrm{p}<0.05$ respectively). On day 70 of the study these values remained the same in both groups. Sixty one patients (43.6\%) who were involved in the study showed the valve structural changes in the internal jugular vein. At the same time we observed congenital pathology of valves manifested by their absence or their underdevelopment and secondary insufficiency (28 (45.9\%) of cases), (7 (11.4\%) of cases). In 2 patients $(3.3 \%)$ the presence of two tier valves of internal jugular veins was revealed. Phlebectasia in combination with reflux was observed in $3(16.7 \%)$ patients with hypertension stage I, in $3(17.6 \%)$ patients with hypertension stage II and 4 (15.4\%) with hypertension stage III. In 19 (13.6\%) patients the veins with hypoplasia (Internal jugular veins had a cross-sectional area less than $2 / 3$ of the cross-sectional area of the common carotid artery) and also small veins were found (cross-sectional area of the internal jugular vein and common carotid artery are equal). On the 70th day of the study all structural changes of the veins remained the same.

While investigating the dynamic pattern of blood flow velocity values of internal jugular veins in patients with hypertension a drop of values in Vmax, Vmed and Vvolmax was found on the internal jugular vein at all stages of the disease to $29.4[14.3 ; 17.6] \mathrm{cm} / \mathrm{s}, 18.7$ [8.9; 26.4] $\mathrm{cm} / \mathrm{s}$ and $421.9[366.9 ; 485.7] \mathrm{ml} / \mathrm{min}$. respectively compared with the control group $(49.4[28.7 ; 68.3] \mathrm{cm} / \mathrm{s}, 23.8[17.6 ; 29.0] \mathrm{cm} / \mathrm{s}$ and 523.9 $[412.0 ; 631.4] \mathrm{ml} / \mathrm{min}$. respectively), $(\mathrm{p}<0.02)$. On 70 day in treatment group 1 restoration of these values at I-III stages of hypertension to the level of control values was noted $(49.4$ [28.7; 68.3], $23.8[17.6 ; 29.0], 523.9$ [412.0; 631.4] respectively, $\mathrm{p}>0.05)$. A similar dynamic pattern was not revealed in treatment group 2 .

While examining vertebral veins $94.1 \%$ of cases had stage I, $96.1 \%$ - had the second stage and $86.8 \%$ of cases had the third stage.

When assessing the dynamic pattern of blood flow velocity values of vertebral veins in patients with hypertension stages I-III the increase of all velocity values was documented in comparison with the control group $(\mathrm{p}<0.04)$. During combined therapy with application of cytoflavin a decrease of values was noted in Vmax, Vmin and Vmed at hypertension stages I-III to the control group values (16.5 [16.0; 16.9], $9.7[9.0 ; 10.3], 24.6[14.6 ; 34.5]$ respectively, $\mathrm{p}>0.05)$. The values remained the same in treatment group 2 .

Thus, with an ultrasonic examination of the intra-and extracranial venous bed at all stages of hypertension the presence of structural 
changes of the valve apparatus of internal jugular veins in $43.6 \%$ of patients was revealed as well as the signs of cerebral venous stasis which are expressed by the velocity values decrease in Rosenthal veins, internal jugular veins and their increase in vertebral veins, a decrease in Rosenthal vein reactivity. During combined cytoflavin therapy, the restoration of linear and volumetric velocity values to control values was noted in Rosenthal veins, internal jugular veins and their decrease in vertebral veins at all stages of the disease.

\section{Discussion and Conclusion}

Hypertensive disease is the cause of severe cardiovascular complications as a result of formation of the heart remodeling and large vessels, including cerebral ones. At the same time, the structural and functional changes that occur in the brain vessels are not that much a compensatory reaction, but the independent cause of further disease progression and independent negative prognostic factor $[8,19]$. In this regard, the search of effective ways of etiopathogenetic impact on the process of vascular remodeling in case of hypertension and elimination of its manifestations is extremely important.

With ultrasonic scanning of the vessels of the arterial bed of the brain structural and functional disorders have been revealed both at the intracranial and extracranial levels in our study. Structural changes were observed in main arteries of the head. These changes were an increase of the thickness of intima media complex and PI in hypertension stage III and in medial cerebral arteries as an IR increase at stage I. In the course of combined therapy with cytoflavin for 70 days structural changes had a persistent character. This suggests that it had no effect on structural changes of the vessels. The decrease in linear and volumetric velocity values observed in the main and intracranial arteries demonstrates a decrease of the arterial inflow to the brain at all stages of hypertension. During combined cytoflavin therapy the restoration of maximum linear blood flow velocity values in common carotid arteries at hypertension stage I, maximum volumetric velocity in internal carotid arteries, velocity values in vertebral arteries at hypertension stages I-II and maximum volumetric velocity in common carotid arteries at all hypertension stages to the level of control values demonstrates its efficacy on blood flow values in vessels of the first structural level at early stages of hypertension progression. While examining the intracranial arterial bed the blood flow depression was found in medial cerebral arteries in all hypertension stages expressed as the reduction of velocity values that also shows arterial flow insufficiency, and in combination with peripheral vessel resistance it shows cerebral perfusion. The restoration of linear blood flow velocity in medial cerebral arteries at stage I only during metabolic therapy is likely to be associated with disorders of small cerebral arteries with progression of arteriosclerosis and arteriopathy with subsequent reduction of sensitivity threshold to therapy at advanced stages. At ultrasonic scanning of the venous bed of the brain s, a decrease in Rosenthal veins reactivity has been found expressed in invariance of the linear blood flow velocity before and after orthostatic loading that is a decrease in hemodynamic reserve and adaptive capacities of the cerebral vascular system. A decrease in all velocity values in orthostasis compared with baseline values during combined therapy with cytoflavin application testified to an adequate reactivity of Rosenthal veins and increase of hemodynamic reserve the state of which in many respects determines prognosis, the choice of treatment and method characterizes the efficiency of the latter. At the beginning of our study we noted cerebral venous haemodynamics disorders at the intracranial and extracranial levels already at hypertension stage I expressed in velocity values decrease compared with control values in Rosenthal veins and internal jugular veins and blood flow increase in the vertebral veins that demonstrates inclusion of the vertebral veins as additional ways of outflow in case of intracranial venous stasis. Results of our research have shown the efficacy of cytoflavin concerning intracranial and extracranial venous hemodynamics in the form of restoration of linear velocity values to control values in Rosenthal veins and internal jugular veins, vertebral veins in recumbent position from stage hypertension I.

Thus, persistent structural changes bear irreversible character, but the effect of the therapy on functional disorders can be explained by its action on endothelium. The studies conducted earlier documented the effect on arterial endothelium at earlier stages of such hypertensive complication as hypertensive encephalopathy. This was characterized by the recovery of arterial hemodynamics at earlier stages. After the correlation analysis between the values of cerebral vessel hemodynamics and vasomotor activity of endothelium had been performed, an assumption was made about a positive influence of therapy on venous endothelium at all stages. This influence is characterized by the recovery of venous hemodynamics in hypertension stages I-III [7]. Thus, a positive effect in relation to functional changes that arise in cerebral vessels can be explained by the effect of cytoflavin on endothelium. This is extremely important, since it is known that the first link of the pathological ischemic cascade triggering all subsequent stages is hypoxia caused by a microcirculation disorder, and changes at the microcirculatory level depend on the condition of the endothelial function determining reactivity of vessels [20-22]. Thus, acting positively on vascular endothelium of intracranial veins, restoring microcirculation the cytoflavin therapy prevents the development of the whole pathological ischemic cascade with the subsequent death of neurons. It is possible to reduce the risk of neurotic complications by blocking the development of changes in the cerebral vascular system and forming cerebral vessel remodeling, and as a sequence to improve life quality and life expectancy of patients with hypertension.

The results obtained during our investigation allow us to recommend cytoflavin for use in a combined therapy of patients with a hypertensive illness from stage I according to the treatment regimen: $10 \mathrm{mg}$ from the 1 st to the 10th day by means of intravenous drop infusion, then 60 days - 2 tablets twice a day.

The presented results have been obtained in the framework of the state assignment of the RF Ministry of Education and Science performed by Ulyanovsk State University.

\section{References}

1. Gusev El, Konovalov AN, Skvortsova VI, Hecht AB (2010) Chronic insufficiency of cerebral blood circulation. Neurology National leaders, GEOTAR-Media, Mascow, Russia.

2. Conradi $A O$ (2012) Key achievements in the combined antihypertensive therapy of the last years. Arterial hypertension 6: 491-496.

3. Almeida RC, Dias DJ, Deguchi KT, Spesia CH, Coelho OR (2015) Prevalence and treatment of hypertension in urban and riverside areas in Porto Velho, the Brazilian Amazon. Postgraduate medicine 127: 66-72.

4. Hermida RC, Ayala DE, Smolensky MH, Fernández JR, Mojón A, et al. (2016) Chronotherapy with conventional blood pressure medications improves management of hypertension and reduces cardiovascular and stroke risks. Hypertension Research 39: 277-292.

5. Wu J, Cheng X, Qu L, Xu T, Zhu G, et al. (2016) Prevalence and Clustering of Major Cardiovascular Risk Factors in China: A Recent Cross-Sectional Survey. Medicine (Baltimore) 95: 2712. 
Citation: Belova LA, Kolotik-Kameneva OY, Mashin VV, Belova NV, Scuderi A, et al. (2016) The Influence of Cytoflavin Therapy on the Cerebral Hemodynamics Condition in Patients with Various Stages of Hypertensive Disease. J Non Invasive Vasc Invest 1: 001.

6. Lesser GT (2016) Neglected Categorical Differences of Hypertension of the Elderly vs. the Young: A Case of Institutional Amnesia. J Am Med Dir Assoc 17: $376-378$.

7. Belova LA, Mashin VV, Kolotik-Kameneva OY, Proshin AN (2014) EFFECT OF THERAPY TSITOFLAVINOM $®$ endothelial function and cerebral hemodynamics in patients with hypertensive encephalopathy. Antibiotics and Chemotherapy 59: 30-36

8. Gulevskaya TS, Morgunov VA (2009) Pathological anatomy of cerebral blood circulation disorders in case of atherosclerosis and arterial hypertension. JSC Publishing House "Meditsina", Russia.

9. Mashin VV, Belova LA, Saprygina LV, Kravchenko MA, Varakin YY, et al (2014) Risk factors for cerebrovascular diseases according to the screening of middle-aged population of Ulyanovsk. The Annals of clinical and experimental neurology 8: 4-9.

10. Suslina ZA, Tanashyan MM, Smirnova IN, et al. (2002) Antioxidant and neurotrophic action of cytoflavin in case of chronic cerbrovascular diseases. Bulletin, St. Petersburg State Academy of Medicine 3: 110-114.

11. Fonyakin AV, Geraskina LA (2011) Modern strategies of secondary prevention of an ischemic stroke. Consilium Medicum 2: 17-22.

12. Belova LA, Kolotik-Kameneva OY, Mashin VV, Saprygin LV, Mashin EV (2014) Efficacy of the energy-modifier cytoflavin in the treatment of patients with hypertensive encephalopathy. Therapeutic archive 86: 65-70.

13. Belova LA, Mashin VV, Nikitin YM, Belova NV, Niyazova RM, et al. (2012) Endothelium dysfunction and condition of structural and functional levels of the vascular system of the brain in case of hypertensive encephalopathy. The Neurologic messenger 1: 3-9
14. Mashin VV, Belova LA, Kadykov AS (2003) Clinical and doppler signs of cerebral venous discirculation in case of hypertensive encephalopathy. The Herald of Practical Neurology. 7: 13-14.

15. Shakhnovich AR, Shakhnovich VA (2009) A noninvasive assessment of venous blood circulation of the brain, CSF circulation and cranial and vertebral volume ratios in case of hydrocephaly. Clinical physiology of blood circulation 3: $5-16$.

16. Caso V, Agnelli G, Paciaroni M (2008) Handbook on cerebral venous thrombosis. In: Bogousslavsky (ed.). Frontiers of neurology and neuroscience (vol 23), Karger Publishers, Basel, Switzerland.

17. Henry-Feugeas MC, Koskas P (2012) Cerebral vascular aging: extending the concept of pulse wave encephalopathy through capillaries to the cerebral veins. Curr Aging Sci 5: 157-67.

18. Shumilina MV, Gorbunova EV (2009) Comprehensive ultrasonic diagnostics of venous outflow disorders. Clinical physiology of blood circulation 3: 21-29.

19. Konradi AO (2008) The HYVET study- the new about the old. Arterial hypertension 14: 397-401.

20. Suslina ZA, Fonyakin AV, Geraskina LA, Trunov ES, Mashin VV, et al. (2010) Practical cardioneurology IMA-Press, Mascow, Russia.

21. Adamson RH, Sarai RK, Altangerel A, Clark JF, Weinbaum S, et al. (2013) Microvascular permeability to water is independent of shear stress, but dependent on flow direction. Am J Physiol Heart Circ Physiol 11: 1077-1084.

22. Mancini A, Serrano-Díaz J, Nava E, D’Alessandro AM, Alonso GL, et al (2014) Crocetin, a carotenoid derived from saffron (Crocus sativus L.), improves acetylcholine-induced vascular relaxation in hypertension. J Vasc Res 51: 393-404. 